\title{
THE NEW FAMILY OF CRACKED SETS AND THE IMAGE SEGMENTATION PROBLEM REVISITED*
}

\author{
MICHEL C. DELFOUR ${ }^{\dagger}$ AND JEAN-PAUL ZOLÉSIO ${ }^{\ddagger}$
}

\author{
Dedicated to Sanjoy Mitter on the occasion of his 70th birthday.
}

\begin{abstract}
The object of this paper is to introduce the new family of cracked sets which yields a compactness result in the $W^{1, p}$-topology associated with the oriented distance function and to give an original application to the celebrated image segmentation problem formulated by Mumford and Shah [21]. The originality of the approach is that it does not require a penalization term on the length of the segmentation and that, within the set of solutions, there exists one with minimum density perimeter as defined by Bucur and Zolésio in [3]. This theory can also handle $N$-dimensional images. The paper is completed with several variations of the problem with or without a penalization term on the length of the segmentation. In particular, it revisits and recasts the earlier existence theorem of Bucur and Zolésio [3] for sets with a uniform bound or a penalization term on the density perimeter in the $W^{1, p}$-framework.
\end{abstract}

1. Introduction. The object of this paper is to introduce the new family of cracked sets which yields a compactness theorem in the $W^{1, p}$-topology associated with the oriented distance function ${ }^{1}$ and to give an original application to the celebrated image segmentation problem formulated by Mumford and Shah [21]. The originality of our approach is that it does not require a penalization term on the length of the segmentation and that, within the set of solutions, there exists one with minimum density perimeter as defined by Bucur and Zolésio in [3]. The paper is completed with several variations of the problem with or without a penalization term on the length of the segmentation. In particular we revisit and recast the earlier existence theorem of [3] in the $W^{1, p}$-framework. The theory is not limited to $2 \mathrm{D}$ problems and can handle $N$-dimensional images. Cracked sets form a very rich family of sets with a huge potential that is not fully exploited in the image segmentation problem. Indeed they can not only be used to partition the frame of an image, but also to detect isolated cracks and points provided an objective function sharper than the one of Mumford and Shah be used. For instance, in view of the connection between image segmentation and fracture theory [1], the theory may have potential applications in problems related to the detection of fractures or cracks or fracture branching and

*Accepted for publication on November 3, 2003. This research has been supported by National Sciences and Engineering Research Council of Canada research grant A-8730 and by a FCAR grant from the Ministère de l'Éducation du Québec.

${ }^{\dagger}$ Centre de recherches mathématiques et Département de mathématiques et de statistique, Université de Montréal, C. P. 6128, succ. Centre-ville, Montréal (Qc), Canada H3C 3J7, E-mail: delfour@CRM.UMontreal.CA

${ }^{\ddagger}$ CNRS and INRIA, INRIA, 2004 route des Lucioles, BP 93, 06902 Sophia Antipolis Cedex, France, E-mail: Jean-Paul.Zolesio@sophia.inria.fr

${ }^{1}$ Also referred to as the algebraic or signed distance function. 
segmentation in geomaterials [2], but this is way beyond the scope of this paper. Some initial considerations about the numerical approximation of cracked sets can be found in [13].

In problems where the shape or the geometry is a design, control, or identification variable, metrics are used to measure the distance between objects, to specify topologies to make sense of continuity and compactness, and to obtain meaningful optimality conditions. From the purely theoretical viewpoint it is now becoming clear that the metric constructed from the $W^{1, p}$-norm ${ }^{2}$ on the oriented distance function (signed or algebraic distance function) is playing a central and natural role in the analysis of such problems. For instance, convergence and compactness in that topology imply the same properties in all other topologies constructed from distance functions to or characteristic functions of a set, its complement, or its boundary. Earlier compactness results using the uniform cone property or the density perimeter or more recent ones using the uniform cusp property also hold in the finer $W^{1, p}$-topology (cf. $[6,7,8]$ and the recent book [11] for an extensive analysis of metrics on subsets of the Euclidean space). In addition, it also plays a key role in other geometric identification problems and the characterization of the space of solutions of the evolution equation of the oriented distance function for initial sets with thin boundary evolving in a velocity field [12].

In $\S 2$ we review the definitions, properties, and the metrics associated with the oriented distance function. In $\S 3$ we review the sets with finite $h$-density perimeter and introduce the new families of cracked sets. We give the associated compactness theorems in the strong $W^{1, p}$-metric topology on the oriented distance function. In $\S 4$ we discuss the formulation ( $\S 4.1$ ) of the $N$-dimensional image segmentation problem and give the main existence theorem for cracked sets without penalization term or bound on the perimeter $(\S 4.2)$. $\S 4.3$ makes use of the $h$-density perimeter which is a relaxation of the $(N-1)$-dimensional upper Minkowski content in two ways: an example of a segmentation whose solution has two open connected parts but an interface of infinite length; and a complement to the existence theorem of $\S 4.2$ by proving that among all the solutions there is one with minimum $h$-density perimeter. In $\S 4.4$ we go back to the formulation of Bucur and Zolésio with respect to the family of sets with a bounded $h$-density perimeter. We give an existence theorem in the case of a uniform bound and no penalization term and in the case of a penalization term and no uniform bound on their perimeter. Finally, as a corollary, we give the existence for those two cases within the family of cracked sets.

In this paper the words set, image, and object will be used equivalently.

Notation 1.1. Given an integer $N \geq 1, \mathrm{~m}_{N}$ and $H_{N-1}$ will denote the $N$ dimensional Lebesgue and (N-1)-dimensional Hausdorff measures. The inner product

\footnotetext{
${ }^{2}$ This topology was introduced in $[9,10]$ and further investigated in [11].
} 
and the norm in $\mathbf{R}^{\mathrm{N}}$ will be written $x \cdot y$ and $|x|$. The complement $\left\{x \in \mathbf{R}^{\mathrm{N}}: x \neq \Omega\right\}$ and the boundary $\bar{\Omega} \cap \overline{\complement \Omega}$ of a subset $\Omega$ of $\mathbf{R}^{\mathrm{N}}$ will be respectively denoted by $\complement \Omega$ or $\mathbf{R}^{\mathrm{N}} \backslash \Omega$ and by $\partial \Omega$ or $\Gamma$. The distance function $d_{A}(x)$ from a point $x$ to a subset $A \neq \varnothing$ of $\mathbf{R}^{\mathrm{N}}$ is defined as $\inf \{|y-x|: y \in A\}$.

\section{Oriented distance function and its properties.}

2.1. Definitions and properties. Given a subset $\Omega$ of $\mathbf{R}^{\mathrm{N}}$ with boundary $\Gamma \neq \varnothing$, the oriented distance function is defined as

$$
b_{\Omega}(x) \stackrel{\text { def }}{=} d_{\Omega}(x)-d_{\complement \Omega}(x) .
$$

There is a one-to-one correspondence between $b_{\Omega}$ and the equivalence class

$$
[\Omega]_{b} \stackrel{\text { def }}{=}\left\{\Omega^{\prime} \subset \mathbf{R}^{\mathrm{N}}: \Gamma^{\prime}=\Gamma \text { and } \overline{\Omega^{\prime}}=\bar{\Omega}\right\}=\left\{\Omega^{\prime} \subset \mathbf{R}^{\mathrm{N}}: b_{\Omega^{\prime}}=b_{\Omega}\right\} .
$$

In general

$$
d_{\bar{\Omega}}=d_{\Omega} \leq d_{\mathrm{int} \Omega} \text { and } d_{\mathrm{Cint} \Omega}=d_{\overline{\mathrm{C} \Omega}}=d_{\mathrm{C} \Omega} \leq d_{\mathrm{C} \bar{\Omega}},
$$

but we only have

$$
b_{\bar{\Omega}} \leq b_{\Omega} \leq b_{\text {int } \Omega} .
$$

where int $\Omega$ and $\bar{\Omega}$ are the interior and closure of $\Omega$. For convex sets we have $b_{\bar{\Omega}}=b_{\Omega}$; for sets verifying the uniform segment property ${ }^{3}$ we have $b_{\bar{\Omega}}=b_{\Omega}=b_{\text {int } \Omega}$. When $\bar{\Omega}$ is a closed submanifold of $\mathbf{R}^{\mathrm{N}}$ of codimension greater or equal to one, then $\bar{\Omega}=\Gamma$ and $b_{\Omega}=d_{\Omega}=d_{\Gamma}$.

The terminology and the notation emphasize the fact that $\nabla b_{\Omega}$ coincides with the exterior normal to the boundary (when it exists). The function $b_{\Omega}$ offers definite conceptual and technical advantages over the function $d_{\Omega}$ and makes it possible to simultaneously deal with open $N$-dimensional subsets and embedded submanifolds of $\mathbf{R}^{\mathrm{N}}$ in the same framework. In the literature, it usually appears as the distance to the boundary $d_{\Gamma}$ with a change of sign across the boundary and is referred to as the algebraic or signed distance function. Definition (2.1) and the associated equivalence classes seem to have been first introduced in 1994 in [9]. The function $b_{\Omega}$ captures many of the geometric properties of the set $\Omega$. For instance, in 1994 it was showed in $[9,11]$ that the property that $\bar{\Omega}$ is convex if and only if $d_{\Omega}$ is convex remains true with $b_{\Omega}$ in place of $d_{\Omega}$.

The function $b_{\Omega}$ is Lipschitz continuous of constant 1 , and $\nabla b_{\Omega}$ exists and $\left|\nabla b_{\Omega}\right| \leq$ 1 almost everywhere in $\mathbf{R}^{\mathrm{N}}$. Thus $b_{\Omega} \in W_{\text {loc }}^{1, p}\left(\mathbf{R}^{\mathrm{N}}\right)$ for all $p, 1 \leq p \leq \infty$. The points

\footnotetext{
${ }^{3} \Omega$ is said to satisfy the uniform segment property if $\exists r>0, \exists \lambda>0$ such that $\forall x \in \Gamma, \exists d \in \mathbf{R}^{\mathrm{N}},|d|=1$,

for which for all $y \in B_{r}(x) \cap \bar{\Omega},(y, y+\lambda d) \subset \operatorname{int} \Omega$.
} 
of $\mathbf{R}^{\mathrm{N}}$ where the gradient of $b_{\Omega}$ does not exist can be divided into two categories: the ones on the boundary $\Gamma$ and the ones outside of $\Gamma$.

Definition 2.1. The set of projections of a point $x \in \mathbf{R}^{\mathrm{N}}$ onto the boundary $\Gamma$ of a set $\Omega, \Gamma \neq \varnothing$,

$$
\Pi_{\Gamma}(x) \stackrel{\text { def }}{=}\left\{p \in \mathbf{R}^{\mathrm{N}}:\left|b_{\Omega}(x)\right|=|p-x|\right\}
$$

since $\left|b_{\Omega}(x)\right|=d_{\Gamma}(x)$; the skeleton of $\Omega$

$$
\operatorname{Sk}(\Omega) \stackrel{\text { def }}{=}\left\{x \in \mathbf{R}^{\mathrm{N}}: \Pi_{\Gamma}(x) \text { is not a singleton }\right\}
$$

(by definition $\operatorname{Sk}(\Omega) \subset \mathbf{R}^{\mathrm{N}} \backslash \Gamma$ ); the set of cracks of $\Omega$

$$
\mathrm{C}(\Omega) \stackrel{\text { def }}{=}\left\{x \in \mathbf{R}^{\mathrm{N}}: \nabla b_{\Omega}^{2}(x) \text { exists but } \nabla b_{\Omega}(x) \text { does not exist }\right\} .
$$

The terminology crack is used here in a very broad sense. $C(\Omega)$ can contain subsets of arbitrary co-dimension. In dimension $N=2$ the corners along a piecewise smooth boundary belong to $\mathrm{C}(\Omega)$. We recall basic properties.

THeOREM 2.1. Let $\Omega$ be a subset of $\mathbf{R}^{\mathrm{N}}$ with $\Gamma \neq \varnothing$.

(i) For all $x \in \Gamma, \nabla b_{\Omega}^{2}(x)$ exists and $\nabla b_{\Omega}^{2}(x)=0$; for all $x \notin \Gamma$

$$
\nabla b_{\Omega}^{2}(x) \text { exists } \Longleftrightarrow \nabla b_{\Omega}(x) \text { exists . }
$$

Hence $\nabla b_{\Omega}(x)$ exists if and only if $x \notin \operatorname{Sk}(\Omega) \cup \mathrm{C}(\Omega)$. Moreover

$$
\operatorname{Sk}(\Omega)=\left\{x \in \mathbf{R}^{\mathrm{N}}: \nabla b_{\Omega}^{2}(x) \text { does not exist }\right\}
$$

and $\operatorname{Sk}(\Omega) \subset \mathbf{R}^{\mathrm{N}} \backslash \Gamma$ and $\mathrm{C}(\Omega) \subset \Gamma$ have zero $\mathrm{m}_{N}$-measure.

(ii) The projection $p_{\Gamma}(x)$ of a point $x \notin \operatorname{Sk}(\Omega)$ onto the boundary $\Gamma$ of $\Omega$ is given in terms of $b_{\Omega}$

$$
p_{\Gamma}(x)=x-\frac{1}{2} \nabla b_{\Omega}^{2}(x)=x-b_{\Omega}(x) \nabla b_{\Omega}(x) .
$$

(iii) The Hadamard semi-derivative $e^{4}$ of $b_{\Omega}^{2}$ always exists

$$
\forall v \in \mathbf{R}^{\mathrm{N}}, \quad d_{H} b_{\Omega}^{2}(x ; v)=2 \min _{p \in \Pi_{\Gamma}(x)}(x-p) \cdot v .
$$

(iv) For all points $x \notin \Gamma$, the Hadamard semiderivative of $b_{\Omega}$ exists and

$$
\forall v \in \mathbf{R}^{\mathrm{N}}, \quad d_{H} b_{\Omega}(x ; v)=\frac{1}{b_{\Omega}(x)} \min _{p \in \Pi_{\Gamma}(x)}(x-p) \cdot v .
$$

For all points $x \in \Gamma, d_{H} b_{\Omega}(x ; v)$ exists if and only if

$$
\forall v \in \mathbf{R}^{\mathrm{N}}, \quad \lim _{t \searrow 0} \frac{b_{\Omega}(x+t v)}{t} \text { exists. }
$$

Proof. (i) and (ii) Cf. [11], Chapter 5, Theorem 4.4 and Chapter $8 \S 5, \S 2, \S 3$, and p. 369). (iii) Cf. [11], Theorem 3.1 (iii), p. 164. (iv) Obvious.

\footnotetext{
${ }^{4}$ A function $f: \mathbf{R}^{\mathrm{N}} \rightarrow \mathbf{R}$ has a Hadamard semi-derivative in $x$ in the direction $v$ if $d_{H} f(x ; v) \stackrel{\text { def }}{=} \lim _{\substack{t \searrow 0 \\ w \rightarrow v}} \frac{f(x+t w)-f(x)}{t}$ exists (cf [11], Chapter 8, Definition 2.1 (ii)).
} 


\subsection{Strong, weak, and uniform metric topologies.}

\subsubsection{Definitions and sets with thin boundary. Definition 2.2.}

(i) The boundary $\Gamma$ of a subset $\Omega$ of $\mathbf{R}^{\mathrm{N}}$ is said to be $\operatorname{thin}^{5}$ if $\mathrm{m}_{N}(\Gamma)=0$; otherwise it is said to be thick.

(ii) Given a nonempty subset $D$ of $\mathbf{R}^{\mathrm{N}}$, define the families

$$
\begin{gathered}
C_{b}(D) \stackrel{\text { def }}{=}\left\{b_{\Omega}: \Omega \subset \bar{D} \text { and } \Gamma \neq \varnothing\right\} \\
C_{b}^{0}(D) \stackrel{\text { def }}{=}\left\{b_{\Omega} \in C_{b}(D): \mathrm{m}_{N}(\Gamma)=0\right\} .
\end{gathered}
$$

The space $C_{b}^{0}(D)$ corresponds to the subfamily of subsets of $\mathbf{R}^{\mathrm{N}}$ with a thin boundary that is a more natural family than the family $C_{b}(D)$ in applications.

2.2.2. Strong metric topologies. In this paper we specialize to the following complete metrics ${ }^{6}$ associated with $b_{\Omega}$ over the subsets of a bounded open hold-all $D$

$$
\begin{aligned}
\rho_{C(D)}\left(\left[\Omega^{\prime}\right],[\Omega]\right) \stackrel{\text { def }}{=}\left\|b_{\Omega^{\prime}}-b_{\Omega}\right\|_{C(\bar{D})}=\max _{x \in \bar{D}}\left|b_{\Omega^{\prime}}(x)-b_{\Omega}(x)\right| \\
\rho_{W^{1, p}(D)}\left(\left[\Omega^{\prime}\right],[\Omega]\right) \stackrel{\text { def }}{=}\left\|b_{\Omega^{\prime}}-b_{\Omega}\right\|_{W^{1, p}(D)} \\
\quad=\left\{\int_{D}\left|b_{\Omega^{\prime}}-b_{\Omega}\right|^{p}+\left|\nabla b_{\Omega^{\prime}}-\nabla b_{\Omega}\right|^{p} d x\right\}^{1 / p} .
\end{aligned}
$$

The space $C_{b}(D)$ is a complete metric space for the metrics (2.10) and (2.11), but the space $C_{b}^{0}(D)$ is complete only with respect to the metric (2.11) (e.g. [11], Chapter 5$)^{7}$. The metric (2.10) is the analogue with $b_{\Omega}$ of the Hausdorff metric defined from $d_{\Omega}$. For the purpose of the paper we shall use the convenient terminology Hausdorff metric, but it should be remembered that this metric with $b_{\Omega}$ is different from the classical Hausdorff metric with $d_{\Omega}$. The $W^{1, p}$-topologies are all equivalent for $1 \leq p<\infty$ (cf. [11] Theorem 5.1, Chapter 5, p. 226).

2.2.3. Weak $W^{1, p}$ and Hausdorff metric topologies. $C_{b}(D)$ is also complete for the weak $W^{1, p}$-topologies which are also all equivalent for $1 \leq p<\infty$. The weak $W^{1, p}$-convergence of sequences of oriented distance functions is equivalent to the strong convergence in the $C(\bar{D})$-topology of uniform convergence (cf. [11] Theorem 5.2 (i)-(ii), Chapter 5, p. 228).

For sets with thin boundaries the strong and weak $W^{1, p}(D)$-convergences of elements of $C_{b}^{0}(D)$ to an element of $C_{b}^{0}(D)$ are equivalent.

Lemma 2.1. Given a bounded open subset $D$ of $\mathbf{R}^{\mathrm{N}}$, let $\left\{\Omega_{n}\right\}$ be a sequence of subsets of $\bar{D}$ such that $\Gamma_{n} \neq \varnothing$ and $\mathrm{m}\left(\Gamma_{n}\right)=0$. Further assume that there exists

\footnotetext{
${ }^{5}$ This terminology is not to be confused with the one of thin set in Capacity Theory.

${ }^{6}$ Other complete metrics can be defined with $d_{\Omega}, d_{\complement \Omega}, d_{\Gamma}$ in place of $b_{\Omega}$.

${ }^{7}$ The completeness of the metric (2.10) is not a trivial consequence of the classical proof in [14] of the completeness of the Hausdorff metric associated with $d_{\Omega}$. To our best knowledge the metrics (2.10) and (2.11) were first introduced by [9] in 1994.
} 
$\Omega \subset \bar{D}$ such that $\Gamma \neq \varnothing$ and $\mathrm{m}(\Gamma)=0$. Then

$$
b_{\Omega_{n}} \rightarrow b_{\Omega} \text { in } W^{1,2}(D) \text {-weak } \Rightarrow b_{\Omega_{n}} \rightarrow b_{\Omega} \text { in } W^{1,2}(D) \text {-strong }
$$

and hence in $W^{1, p}(D)$-strong for all $p, 1 \leq p<\infty$.

Proof. Same proof as in part (ii) of the proof of Theorem 10.1 in [11]. Since, for all $n \geq 1, \mathrm{~m}\left(\Gamma_{n}\right)=0=\mathrm{m}(\Gamma),\left|\nabla b_{\Omega}\right|=1=\left|\nabla b_{\Omega_{n}}\right|$ almost everywhere in $D$ (cf. [11], Theorem 3.2, p. 215). As a result

$$
\begin{aligned}
& \int_{D}\left|\nabla b_{\Omega_{n}}-\nabla b_{\Omega}\right|^{2} d x=\int_{D}\left|\nabla b_{\Omega_{n}}\right|^{2}+\left|\nabla b_{\Omega}\right|^{2}-2 \nabla b_{\Omega_{n}} \cdot \nabla b_{\Omega} d x \\
& =2 \int_{D}\left(1-\nabla b_{\Omega_{n}} \cdot \nabla b_{\Omega}\right) d x \rightarrow 2 \int_{D}\left(1-\left|\nabla b_{\Omega}\right|^{2}\right) d x=2 \int_{D} \chi_{\Gamma} d x=0 .
\end{aligned}
$$

Therefore $\nabla b_{\Omega_{n}} \rightarrow \nabla b_{\Omega}$ in $L^{2}(D)^{N}$-strong and $b_{\Omega_{n}} \rightarrow b_{\Omega}$ in $W^{1,2}(D)$-strong, since the convergence $b_{\Omega_{n}} \rightarrow b_{\Omega}$ in $L^{2}(D)$-strong follows from the weak convergence in $W^{1,2}(D)$. The convergence in $W^{1, p}(D)$-strong follows from the equivalence of the topologies on $C_{b}(D)$ (cf. [11]. Chapter 5, Theorem 5.1 (i)).

2.2.4. Other metric topologies. The following theorem is central. It shows that convergence and compactness in the metric $\rho_{W^{1, p}(D)}$ will imply the same properties in all other topologies (cf. [11], Theorem 5.1, Chapter 5, p. 226). Recall that $b_{\Omega}^{+}=d_{\Omega}, b_{\Omega}^{-}=d_{\mathrm{C} \Omega}$, and $\left|b_{\Omega}\right|=d_{\Gamma}$, and that $\chi_{\mathrm{int} \Omega}=\left|\nabla d_{\complement \Omega}\right|, \chi_{\mathrm{int} \mathrm{C} \Omega}=\left|\nabla d_{\Omega}\right|$, and $\chi_{\Gamma}=1-\left|\nabla d_{\Gamma}\right|$ a.e. in $\mathbf{R}^{\mathrm{N}}$.

TheOrem 2.2. Let $D$ be a bounded open subset of $\mathbf{R}^{\mathrm{N}}$. The map

$$
b_{\Omega} \mapsto\left(b_{\Omega}^{+}, b_{\Omega}^{-},\left|b_{\Omega}\right|\right)=\left(d_{\Omega}, d_{\complement \Omega}, d_{\partial \Omega}\right): C_{b}(D) \subset W^{1 \cdot p}(D) \rightarrow W^{1 \cdot p}(D)^{3}
$$

and for all $p, 1 \leq p<\infty$, the map

$$
b_{\Omega} \mapsto\left(\chi_{\partial \Omega}, \chi_{\mathrm{int} \Omega}, \chi_{\mathrm{int} \complement \Omega}\right): W^{1, p}(D) \rightarrow L^{p}(D)^{3}
$$

are continuous.

3. Some families of sets. In this section we review families of sets and their properties that will be used in the paper: the sets with a finite density perimeter and the new cracked sets. We give the main associated compactness theorems to deal with the existence of minimizing solutions in $\S 4$.

Notation 3.1. Given $h>0$ the open and closed tubular neighborhoods of a set $A$ are defined as

$$
U_{h}(A) \stackrel{\text { def }}{=}\left\{x \in \mathbf{R}^{\mathrm{N}}: d_{A}(x)<h\right\} \quad A_{h} \stackrel{\text { def }}{=}\left\{x \in \mathbf{R}^{\mathrm{N}}: d_{A}(x) \leq h\right\} .
$$

Recalling that $d_{\Gamma}(x)=\left|b_{\Omega}(x)\right|$ we also have $U_{h}(\Gamma)=\left\{x \in \mathbf{R}^{\mathrm{N}}:\left|b_{\Omega}(x)\right|<h\right\}$. 
3.1. Sets with finite density perimeter. This family of sets introduced in 1996 by Bucur and Zolésio [3] is based on a relaxation of the $(N-1)$-dimensional upper Minkowski content which leads to the compactness Theorem 3.1. We recall the definition and give the proof of the compactness for the $W^{1, p}$-topology under a uniform bound on the $h$-density perimeter.

Definition 3.1. Let $h>0$ be a fixed real and $\Omega$ a subset of $\mathbf{R}^{\mathrm{N}}$ with nonempty boundary $\Gamma$. Consider the quotient

$$
P_{h}(\Gamma) \stackrel{\text { def }}{=} \sup _{0<k<h} \frac{\mathrm{m}_{N}\left(U_{k}(\Gamma)\right)}{2 k} .
$$

Whenever $P_{h}(\Gamma)$ is finite, we say that $\Omega$ has a finite $h$-density perimeter.

It was shown in [3] that, whenever $P_{h}(\Gamma)$ is finite, for all $0<k<h, \Gamma \subset$ $U_{k}(\Gamma)$ and $\mathrm{m}(\Gamma) \leq \mathrm{m}\left(U_{k}(\Gamma)\right) \leq k c$. By letting $k$ go to zero we get $\mathrm{m}(\Gamma)=0$. The compactness result of [3] can now be sharpened and recast in the $W^{1, p}$-topology from which convergence in all other topologies of Theorem 2.2 follows. We also recover the lower semicontinuity of the $h$-density perimeter.

Theorem 3.1. Let $D \neq \varnothing$ be a bounded open subset of $\mathbf{R}^{\mathrm{N}}$ and $\left\{\Omega_{n}\right\}, \Gamma_{n} \neq \varnothing$, be a sequence of subsets of $\bar{D}$. Assume that

$$
\exists h>0 \text { and } c>0 \text { such that } \forall n, \quad P_{h}\left(\Gamma_{n}\right) \leq c .
$$

Then there exist a subsequence $\left\{\Omega_{n_{k}}\right\}$ and a subset $\Omega, \Gamma \neq \varnothing$, of $\bar{D}$ such that

$$
\begin{gathered}
P_{h}(\Gamma) \leq \liminf _{n \rightarrow \infty} P_{h}\left(\Gamma_{n}\right) \leq c \\
\forall \bar{p}, 1 \leq \bar{p}<\infty, \quad b_{\Omega_{n_{k}}} \rightarrow b_{\Omega} \text { in } W^{1, \bar{p}}\left(U_{h}(D)\right) \text {-strong. }
\end{gathered}
$$

Proof. The proof essentially rests on Lemmas 2.1 and the fact that $P_{h}(\Gamma) \leq c$ implies $\mathrm{m}_{N}(\Gamma)=0$. Since $D$ is bounded, the family of oriented distance functions $C_{b}(D)$ is compact in $C(\bar{D})$ and $W^{1, p}(D)$-weak for all $p, 1 \leq p<\infty$ (cf. [11], Theorem 2.2 (ii), p. 210, and Theorem 5.2 (iii), p. 228). So there exist $b_{\Omega} \in C_{b}(D)$ and a subsequence, still indexed by $n$, such that $b_{\Omega_{n}} \rightarrow b_{\Omega}$ in the above topologies. Moreover, for all $k, 0<k<h$, and all $\varepsilon, 0<\varepsilon<h-k$,

$$
\exists N(\varepsilon)>0 \text { such that } \forall n \geq N(\varepsilon), \quad U_{k-\varepsilon}\left(\Gamma_{n}\right) \subset U_{k}(\Gamma) \subset U_{k+\varepsilon}\left(\Gamma_{n}\right)
$$

(cf. proof of part (i) of Theorem 9.2 in [11], p. 251). As a result for all $n \geq N(\varepsilon)$,

$$
\begin{gathered}
\frac{\mathrm{m}_{N}\left(U_{k-\varepsilon}\left(\Gamma_{n}\right)\right)}{2(k-\varepsilon)} \frac{k-\varepsilon}{k} \leq \frac{\mathrm{m}_{N}\left(U_{k}(\Gamma)\right)}{2 k} \leq \frac{\mathrm{m}_{N}\left(U_{k+\varepsilon}\left(\Gamma_{n}\right)\right)}{2(k+\varepsilon)} \frac{k+\varepsilon}{k} \leq c \frac{k+\varepsilon}{k} \\
\Rightarrow \frac{\mathrm{m}_{N}\left(U_{k}(\Gamma)\right)}{2 k} \leq \frac{\mathrm{m}_{N}\left(U_{k+\varepsilon}\left(\Gamma_{n}\right)\right)}{2(k+\varepsilon)} \frac{k+\varepsilon}{k} \leq P_{h}\left(\Gamma_{n}\right) \frac{k+\varepsilon}{k} \\
\Rightarrow \frac{\mathrm{m}_{N}\left(U_{k}(\Gamma)\right)}{2 k} \leq \liminf _{n \rightarrow \infty} P_{h}\left(\Gamma_{n}\right) \frac{k+\varepsilon}{k} .
\end{gathered}
$$


Going to the limit as $\varepsilon$ goes to zero in the second and fourth terms

$$
\begin{gathered}
\forall k, 0<k<h, \quad \frac{\mathrm{m}_{N}\left(U_{k}(\Gamma)\right)}{2 k} \leq \liminf _{n \rightarrow \infty} P_{h}\left(\Gamma_{n}\right) \leq c \\
\Rightarrow P_{h}(\Gamma) \leq \liminf _{n \rightarrow \infty} P_{h}\left(\Gamma_{n}\right) \leq c \Rightarrow \mathrm{m}_{N}(\Gamma)=0 .
\end{gathered}
$$

The theorem now follows from the fact that $\mathrm{m}_{N}(\Gamma)=0$ and Lemma 2.1.

Corollary 3.1. Let $D \neq \varnothing$ be a bounded open subset of $\mathbf{R}^{\mathrm{N}}$ and $\Omega, \Gamma \neq \varnothing$, be a subset of $\bar{D}$ such that

$$
\exists h>0 \text { and } c>0 \text { such that } P_{h}(\Gamma) \leq c .
$$

Then the mapping $b_{\Omega^{\prime}} \rightarrow P_{h}\left(\Gamma^{\prime}\right): C_{b}(D) \rightarrow \mathbf{R} \cup\{+\infty\}$ is lower semicontinuous in $\Omega$ for the $W^{1, p}(D)$-topology.

Proof. Since we have a metric topology, it is sufficient to prove the property for $W^{1, p}(D)$-converging sequences $\left\{b_{\Omega_{n}}\right\}$ to $b_{\Omega}$. From that point on the argument is the same as the one used to get (3.6) in the proof of Theorem 3.1 after the extraction of the subsequence.

REMARK 3.1. It is important to notice that even if $\left\{\Omega_{n}\right\}$ is a $W^{1, p}$-convergent sequence of bounded open subsets of $\mathbf{R}^{\mathrm{N}}$ with a uniformly bounded perimeter, the limit set $\Omega$ need not be an open set or have a nonempty interior int $\Omega$ such that $b_{\Omega}=b_{\text {int } \Omega}$. It would be tempting to say that $b_{\Omega_{n}} \rightarrow b_{\Omega}$ implies $d_{\complement_{\Omega_{n}}} \rightarrow d_{\complement \Omega}$ and use the open set $\operatorname{int} \Omega=\complement \bar{\complement} \Omega$ for which $d_{\complement \Omega}=d_{\complement \text { int } \Omega}$ to conclude that $b_{\Omega}=b_{\text {int } \Omega}$. This is incorrect as can be seen on the following example shown in Figure 1. Consider a family $\left\{\Omega_{n}\right\}$ of
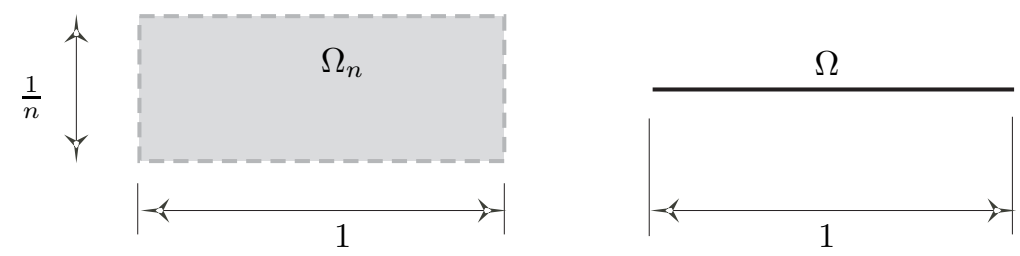

FIG. 1. $W^{1, p}$-convergence of a sequence of open subsets $\left\{\Omega_{n}: n \geq 1\right\}$ of $\mathbf{R}^{2}$ with uniformly bounded density perimeter to a set with empty interior

open rectangles in $\mathbf{R}^{2}$ of width equal to 1 and height $1 / n, n \geq 1$, an integer. Their density perimeter is bounded by 4 and the $b_{\Omega_{n}}$ 's converge to $b_{\Omega}$ for $\Omega$ equal to the line of length 1 which has no interior.

3.2. The new families of cracked sets. In this section we introduce new families of thin sets which are well-suited for image segmentation. They are more general than sets which are locally the epigraph of a continuous function in the sense that they include domains with cracks, and sets that can be made up of components of different co-dimensions. The Hausdorff $(N-1)$ measure of their boundary is not 
necessarily finite. Yet compact families (in the $W^{1, p}$-topology) of such sets can be constructed.

First recall the following definitions of the liminf and limsup for the following differential quotient of a function $f: V(x) \subset \mathbf{R}^{\mathrm{N}} \rightarrow \mathbf{R}$ defined in a neighborhood $V(x)$ of a point $x \in \mathbf{R}^{\mathrm{N}}$ in the direction $d \in \mathbf{R}^{\mathrm{N}}$

$$
\begin{aligned}
& \liminf _{t \searrow 0} \frac{f(x+t d)-f(x)}{t} \stackrel{\text { def }}{=} \lim _{\delta \searrow 0} \inf _{0<t<\delta} \frac{f(x+t d)-f(x)}{t} \\
& \limsup _{t \searrow 0} \frac{f(x+t d)-f(x)}{t} \stackrel{\text { def }}{=} \lim _{\delta \searrow 0} \sup _{0<t<\delta} \frac{f(x+t d)-f(x)}{t} \\
& \liminf _{\substack{t \backslash 0 \\
w \rightarrow d}} \frac{f(x+t w)-f(x)}{t} \stackrel{\text { def }}{=} \lim _{\delta \searrow 0} \inf _{\substack{0<t<\delta \\
|w-d|_{\mathrm{R} N}<\delta \\
(t, w) \neq(0, v)}} \frac{f(x+t w)-f(x)}{t} \\
& \limsup _{\substack{t \searrow 0 \\
w \rightarrow d}} \frac{f(x+t w)-f(x)}{t} \stackrel{\text { def }}{=} \lim _{\delta \searrow 0} \sup _{\substack{0<t<\delta \\
0<-\left.d\right|_{\mathbf{R}}<\delta \\
(t, w) \neq(0, v)}} \frac{f(x+t w)-f(x)}{t} .
\end{aligned}
$$

They are lower and upper semiderivatives ${ }^{8}$ of the Dini type. However we shall not introduce a new notation since the liminf and limsup are more explicit.

DEFINITION 3.2.

(i) A set $\Omega$ in $\mathbf{R}^{\mathrm{N}}, \Gamma \neq \varnothing$, is said to be weakly cracked if

$$
\forall x \in \Gamma, \exists d \in \mathbf{R}^{\mathrm{N}},|d|=1 \text {, such that } \limsup _{\substack{t \searrow 0 \\ w \rightarrow d}} \frac{d_{\Gamma}(x+t w)}{t}>0 .
$$

(ii) A set $\Omega$ in $\mathbf{R}^{\mathrm{N}}, \Gamma \neq \varnothing$, is said to be cracked if

$$
\forall x \in \Gamma, \exists d \in \mathbf{R}^{\mathrm{N}},|d|=1, \text { such that } \liminf _{t \searrow 0} \frac{d_{\Gamma}(x+t d)}{t}>0 .
$$

(iii) A set $\Omega$ in $\mathbf{R}^{\mathrm{N}}, \Gamma \neq \varnothing$, is said to be strongly cracked ${ }^{9}$ if

$$
\forall x \in \Gamma, \exists d \in \mathbf{R}^{\mathrm{N}},|d|=1 \text {, such that } \liminf _{t \rightarrow 0} \frac{d_{\Gamma}(x+t d)}{|t|}>0 .
$$

Strongly cracked implies cracked, and cracked implies weakly cracked since

$$
\begin{aligned}
\liminf _{\substack{t \backslash 0 \\
w \rightarrow d}} \frac{d_{\Gamma}(x+t w)}{t} & \leq \liminf _{t \backslash 0} \frac{d_{\Gamma}(x+t d)}{t} \\
& \leq \limsup _{t \searrow 0} \frac{d_{\Gamma}(x+t d)}{t} \leq \limsup _{\substack{t \backslash 0 \\
w \rightarrow d}} \frac{d_{\Gamma}(x+t w)}{t} .
\end{aligned}
$$

\footnotetext{
${ }^{8}$ Note that the $(t, d), 0<t<\delta$, is allowed in the inf and the sup of the last two definitions and the constraint $(t, w) \neq(0, v)$ can be removed.

${ }^{9}$ Here the definition is

$$
\liminf _{t \rightarrow 0} \frac{f(x+t d)-f(x)}{|t|} \stackrel{\text { def }}{=} \lim _{\delta \searrow 0} \inf _{0<|t|<\delta} \frac{f(x+t d)-f(x)}{t} .
$$
}


The special terminology of Definition 3.2 is motivated by the fact that the boundary of such a set has zero $N$-dimensional Lebesgue measure (cf. Lemma 3.1) and can be made up of cusps, points, cracks or hairs as shown in Figure 2. This terminology is

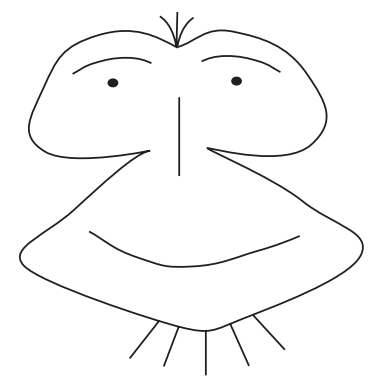

FIG. 2. Example of a two-dimensional strongly cracked set

introduced here to provide an intuitive description of the sets.

The weakly cracked property is verified in any point of the boundary where the gradient of $d_{\Gamma}$ does not exist; in boundary points where the gradient exists it is not identically 0 . This is a very large family of sets that includes domains which are locally the epigraph of a continuous function. There are obvious variations of the above definitions and the forthcoming compactness Theorem 3.2 by replacing $d_{\Gamma}$ by $d_{\Omega}, d_{\complement \Omega}$ or $b_{\Omega}$.

Lemma 3.1. Let $\Omega, \Gamma \neq \varnothing$, be a subset of $\mathbf{R}^{\mathrm{N}}, x \in \Gamma$, and $d,|d|=1$, be a direction in $\mathbf{R}^{\mathrm{N}}$. If the semiderivative $d d_{\Gamma}(x ; d)$ does not exist then

$$
\limsup _{\substack{t \searrow 0 \\ w \rightarrow d}} \frac{d_{\Gamma}(x+t w)}{t}>0 .
$$

Proof. Since the function $d_{\Gamma}$ is Lipschitzian, the limit of the quotient

$$
d d_{\Gamma}(x ; d) \stackrel{\text { def }}{=} \lim _{t \searrow 0} \frac{d_{\Gamma}(x+t d)-d_{\Gamma}(x)}{t}
$$

exists if and only if the limit of the quotient

$$
\lim _{\substack{t \searrow 0 \\ w \rightarrow d}} \frac{d_{\Gamma}(x+t w)-d_{\Gamma}(x)}{t}
$$

exists (cf [11], Chapter 8, Theorem 2.1(i)). Moreover, by the Lipschitz continuity,

$$
\left|\frac{d_{\Gamma}(x+t w)-d_{\Gamma}(x)}{t}\right| \leq\left|\frac{t w}{t}\right|=|w| \rightarrow|d|
$$

and hence the liminf and the limsup of the quotient exist and are finite.

For $x \in \Gamma d_{\Gamma}(x)=0$. Therefore $d d_{\Gamma}(x ; d)$ does not exists if and only if

$$
\limsup _{\substack{t \searrow 0 \\ w \rightarrow d}} \frac{d_{\Gamma}(x+t w)}{t}>\liminf _{\substack{t \searrow 0 \\ w \rightarrow d}} \frac{d_{\Gamma}(x+t w)}{t} \geq 0
$$


since the last term is nonnegative. This completes the proof.

Theorem 3.2.

(i) A weakly cracked set $\Omega$ is thin, that is $\mathrm{m}(\Gamma)=0$, and

$$
\Gamma \subset \overline{\operatorname{int} \Omega} \cup \overline{\operatorname{int} \complement \Omega} \text {. }
$$

Moreover in any point $x \in \Gamma$ either $\nabla b_{\Omega}(x)$ exists and is different from zero or $\nabla b_{\Omega}(x)$ does not exists (set of cracks).

(ii) Given a cracked set $\Omega$, for each $x \in \Gamma$ there exists a direction $d \in \mathbf{R}^{\mathrm{N}},|d|=1$, such that

$$
1 \geq \underline{\ell}(x) \stackrel{\text { def }}{=} \liminf _{t \searrow 0} \frac{d_{\Gamma}(x+t d)}{t}>0
$$

and for all $\varepsilon, 0<\varepsilon<\underline{\ell}(x)$, there exists $\delta>0$ such that

$$
\begin{gathered}
\forall t, 0<t<\delta, \quad d_{\Gamma}(x+t d) \geq(\underline{\ell}(x)-\varepsilon) t \\
\Rightarrow x+C(\delta \cos \omega, \omega, d) \subset \mathbf{R}^{\mathrm{N}} \backslash \Gamma, \quad \sin \omega=\underline{\ell}(x)-\varepsilon, 0<\omega \leq \pi / 2
\end{gathered}
$$

$(C(\lambda, \omega, d)$ is the open cone in 0 of direction $d$, height $\lambda$ and aperture $\omega)$.

(iii) Given a strongly cracked set $\Omega$, for each $x \in \Gamma$ there exists a direction $d \in \mathbf{R}^{\mathrm{N}}$, $|d|=1$, such that

$$
1 \geq \underline{\ell}(x) \stackrel{\text { def }}{=} \liminf _{t \rightarrow 0} \frac{d_{\Gamma}(x+t d)}{|t|}>0
$$

and for all $\varepsilon, 0<\varepsilon<\underline{\ell}(x)$, there exists $\delta>0$ such that

$$
\begin{gathered}
\forall t, 0<|t|<\delta, \quad d_{\Gamma}(x+t d) \geq(\underline{\ell}(x)-\varepsilon)|t| \\
\Rightarrow x \pm C(\delta \cos \omega, \omega, d) \subset \mathbf{R}^{\mathrm{N}} \backslash \Gamma, \quad \sin \omega=\underline{\ell}(x)-\varepsilon, 0<\omega \leq \pi / 2 .
\end{gathered}
$$

Proof. (i) We already know that $\nabla d_{\Gamma}$ exists almost everywhere in $\mathbf{R}^{\mathrm{N}}$ and that, whenever it exists,

$$
\left|\nabla d_{\Gamma}(x)\right|= \begin{cases}0, & \text { if } x \in \Gamma \\ 1, & \text { if } x \notin \Gamma\end{cases}
$$

(cf. [11], Chapter 4, Theorem 3.2 (i)). Therefore if $\nabla d_{\Gamma}(x)$ exists in a point $x \in \Gamma$, $\nabla d_{\Gamma}(x)=0$ and for all $d,|d|=1$,

$$
\limsup _{\substack{t \searrow 0 \\ w \rightarrow d}} \frac{d_{\Gamma}(x+t w)}{t}=\limsup _{\substack{t \searrow 0 \\ w \rightarrow d}} \frac{d_{\Gamma}(x+t w)-d_{\Gamma}(x)}{t}=\nabla d_{\Gamma}(x) \cdot d=0
$$

which contradicts the weakly cracked property. Hence the points of $\Gamma$ are points where $\nabla d_{\Gamma}(x)$ does not exist which is itself a set of zero measure. Moreover, if $\nabla b_{\Omega}(x)$ exists in a point $x \in \partial \Omega$, then $b_{\Omega}(x)=0$ and for all $d,|d|=1$,

$$
\lim _{\substack{t \searrow 0 \\ w \rightarrow d}} \frac{b_{\Omega}(x+t w)}{t}=\lim _{\substack{t \searrow 0 \\ w \rightarrow d}} \frac{b_{\Omega}(x+t w)-b_{\Omega}(x)}{t}=\nabla b_{\Omega}(x) \cdot d .
$$


If $\nabla b_{\Omega}(x)=0$, then for all $d$

$$
\begin{gathered}
\lim _{\substack{t \searrow 0 \\
w \rightarrow d}} \frac{b_{\Omega}(x+t w)}{t}=\lim _{\substack{t \searrow 0 \\
w \rightarrow d}} \frac{b_{\Omega}(x+t w)-b_{\Omega}(x)}{t}=\nabla b_{\Omega}(x) \cdot d=0 \\
\Rightarrow \lim _{\substack{t \searrow 0 \\
w \rightarrow d}} \frac{d_{\Gamma}(x+t w)}{t}=\lim _{\substack{t \searrow 0 \\
w \rightarrow d}}\left|\frac{b_{\Omega}(x+t w)}{t}\right|=0
\end{gathered}
$$

and $\nabla d_{\partial \Omega}(x)=0$ which contradicts our assumption. Therefore if $\nabla b_{\Omega}(x)$ exists in a point $x \in \partial \Omega, \nabla b_{\Omega}(x) \neq 0$. For any $x \in \Gamma$ introduce the notation

$$
\bar{\ell}(x) \stackrel{\text { def }}{=} \limsup _{\substack{t \searrow 0 \\ w \rightarrow d}} \frac{d_{\Gamma}(x+t w)}{t} .
$$

By assumption $\bar{\ell}(x)>0$ and for all $\delta>0$

$$
\bar{\ell}(x) \leq \sup _{\substack{0<t<\delta \\|w-d|<\delta}} \frac{d_{\Gamma}(x+t w)}{t}
$$

Hence there exist sequences $\left\{t_{n}\right\}, t_{n} \rightarrow 0$, and $\left\{w_{n}\right\}, w_{n} \rightarrow d$, such that

$$
0<\bar{\ell}(x) / 2 \leq \frac{d_{\Gamma}\left(x+t_{n} w_{n}\right)}{t} \Rightarrow \mathbf{R}^{\mathrm{N}} \backslash \Gamma \ni x_{n} \stackrel{\text { def }}{=} x+t_{n} w_{n} \rightarrow x
$$

and necessarily $\Gamma \subset \overline{\operatorname{int} \Omega \cup \operatorname{int} \complement \Omega} \subset \overline{\operatorname{int} \Omega} \cup \overline{\operatorname{int} \complement \Omega}$.

(ii) Given a cracked set $\Omega$, for each $x \in \Gamma$ there exists a direction $d \in \mathbf{R}^{\mathrm{N}},|d|=1$, such that

$$
\underline{\ell}(x) \stackrel{\text { def }}{=} \liminf _{t \searrow 0} \frac{d_{\Gamma}(x+t d)}{t}>0
$$

and for all $\varepsilon, 0<\varepsilon<\underline{\ell}(x)$, there exists $\delta>0$ such that

$$
\forall t, 0<t<\delta, \quad d_{\Gamma}(x+t d) \geq(\underline{\ell}(x)-\varepsilon) t .
$$

Recall that since $d_{\Gamma}$ is Lipschizian of constant one, we necessarily have $1 \geq \underline{\ell}(x)$ for $|d|=1$. Therefore

$$
x+C(\delta \cos \omega, \omega, d) \subset \mathbf{R}^{\mathrm{N}} \backslash \Gamma, \quad \sin \omega=\underline{\ell}(x)-\varepsilon, 0<\omega \leq \pi / 2 .
$$

(iii) Similar to the proof of part (ii).

TheOREM 3.3. Let $D$ be a bounded open subset of $\mathbf{R}^{\mathrm{N}}$ and $\alpha>0$ and $h>0$ be 
real numbers ${ }^{10}$. Consider the families

$$
\begin{aligned}
& \mathcal{F}(D, h, \alpha) \stackrel{\text { def }}{=}\left\{\begin{aligned}
& \Gamma \neq \varnothing \text { and } \forall x \in \Gamma, \exists d,|d|=1, \\
\Omega \subset \bar{D}: & \text { such that } \inf _{0<t<h} \frac{d_{\Gamma}(x+t d)}{t} \geq \alpha
\end{aligned}\right\} \\
& C_{b}^{h, \alpha}(D) \stackrel{\text { def }}{=}\left\{b_{\Omega}: \Omega \in \mathcal{F}(D, h, \alpha)\right\} .
\end{aligned}
$$

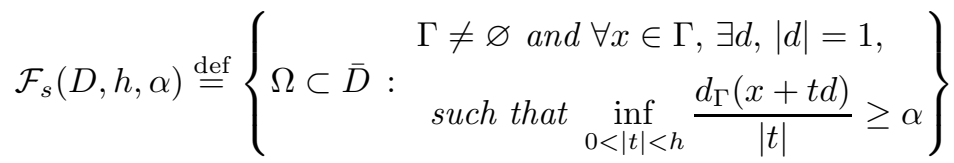

$$
\begin{aligned}
& \left(C_{b}^{h, \alpha}\right)_{s}(D) \stackrel{\text { def }}{=}\left\{b_{\Omega}: \Omega \in \mathcal{F}_{s}(D, h, \alpha)\right\} .
\end{aligned}
$$

Then $C_{b}^{h, \alpha}(D)$ and $\left(C_{b}^{h, \alpha}\right)_{s}(D)$ are compact in $W^{1, p}(D), 1 \leq p<\infty$.

Proof. (i) The family $C_{b}^{h, \alpha}(D)$ is contained in $C_{b}(D)$ which is compact in the uniform topology of $C(\bar{D})$ and in the weak topology of $W^{1, p}(D), 1 \leq p<\infty$ (cf. [11], Chapter 4, Theorem 2.2 (ii), p. 210 and Theorem 5.2 (iii). p. 228). Therefore, given a sequence $\left\{b_{\Omega_{n}}\right\}$ in $C_{b}^{h, \alpha}(D)$, there exist a subsequence, still denoted $\left\{b_{\Omega_{n}}\right\}$, and $b_{\Omega} \in C_{b}(D)$ such that $b_{\Omega_{n}} \rightarrow b_{\Omega}$ in $W^{1, p}(D)$-weak and $C(\bar{D})$. In addition, by definition of the elements of $C_{b}^{h, \alpha}(D)$, condition (3.9) is verified and each $\Omega_{n}$ has thin boundary. We want to show that $b_{\Omega} \in C_{b}^{h, \alpha}(D)$. Once this is proven, from Theorem 3.2 (i), $\Omega$ has a thin boundary. Hence the weak $W^{1, p}(D)$ convergence implies the strong $W^{1, p}(D)$ convergence by Lemma 2.1. In view of the continuity of the map $b_{\Omega} \rightarrow d_{\Gamma}=\left|b_{\Omega}\right|: C(\bar{D}) \rightarrow C(\bar{D}), d_{\Gamma_{n}} \rightarrow d_{\Gamma}$ in $C(\bar{D})$. From Lemma 10.1 in Chapter 5 of [11], given $x \in \Gamma$, there exists a subsequence of $\left\{b_{\Omega_{n}}\right\}$, still denoted $\left\{b_{\Omega_{n}}\right\}$, and for each $n \geq 1$ points $x_{n} \in \Gamma_{n} \cap B(x, 1 / n)$. Hence $x_{n} \rightarrow x$. By assumption

$$
\forall n \geq 1, \exists d_{n} \in \mathbf{R}^{\mathrm{N}},\left|d_{n}\right|=1 \text {, such that } \inf _{0<t<h} \frac{d_{\Gamma_{n}}\left(x_{n}+t d_{n}\right)}{t} \geq \alpha .
$$

Since the $d_{n}$ 's have norm one, there exist a subsequence, still denoted $\left\{d_{n}\right\}$, and $d$, $|d|=1$, such that $d_{n} \rightarrow d$. Fix $t, 0<t<\delta$. Given $\varepsilon>0$, there exists $N$ such that for all $n \geq N$

$$
\left|x_{n}-x\right|<\varepsilon t, \quad\left|d_{n}-d\right|<\delta_{n}<\varepsilon, \quad\left\|d_{\Gamma_{n}}-d_{\Gamma}\right\|_{C(\bar{D})}<\varepsilon t .
$$

\footnotetext{
${ }^{10}$ In view of the fact that the distance function $d_{\Gamma}$ is Lipschitzian with constant 1 , we necessarily have $0<\alpha \leq 1$.
} 
Fix $n=N$ and consider the following estimates

$$
\begin{aligned}
& \frac{d_{\Gamma}(x+t d)}{t} \\
\geq & \frac{d_{\Gamma_{n}}\left(x_{n}+t d_{n}\right)}{t}-\frac{\left|d_{\Gamma_{n}}\left(x+t d_{n}\right)-d_{\Gamma_{n}}\left(x_{n}+t d\right)\right|}{t} \\
& \quad-\frac{\left|d_{\Gamma}(x+t d)-d_{\Gamma}\left(x_{n}+t d\right)\right|}{t}-\frac{\left|d_{\Gamma}\left(x_{n}+t d\right)-d_{\Gamma_{n}}\left(x_{n}+t d\right)\right|}{t} \\
\geq & \alpha-\varepsilon-\left|d-d_{n}\right|-\frac{\left|x-x_{n}\right|}{t}-\frac{|| d_{\Gamma}-d_{\Gamma_{n}} \|_{C \bar{D})}}{t} \geq \alpha-4 \varepsilon \\
\Rightarrow & \forall \varepsilon>0, \inf _{0<t<h} \frac{d_{\Gamma}(x+t d)}{t} \geq \alpha-4 \varepsilon \quad \Rightarrow \inf _{0<t<h} \frac{d_{\Gamma}(x+t d)}{t} \geq \alpha .
\end{aligned}
$$

Therefore $b_{\Omega} \in C_{b}^{h, \alpha}(D)$ and this completes the proof of the compactness.

(ii) The proof for $\left(C_{b}^{h, \alpha}\right)_{s}(D)$ is identical with obvious changes.

\section{The segmentation of $N$-dimensional images.}

4.1. Problem formulation. Typically the image segmentation functional of Mumford and Shah [21] aims at identifying two dimensional objects in a two dimensional frame as shown in Figure 3.

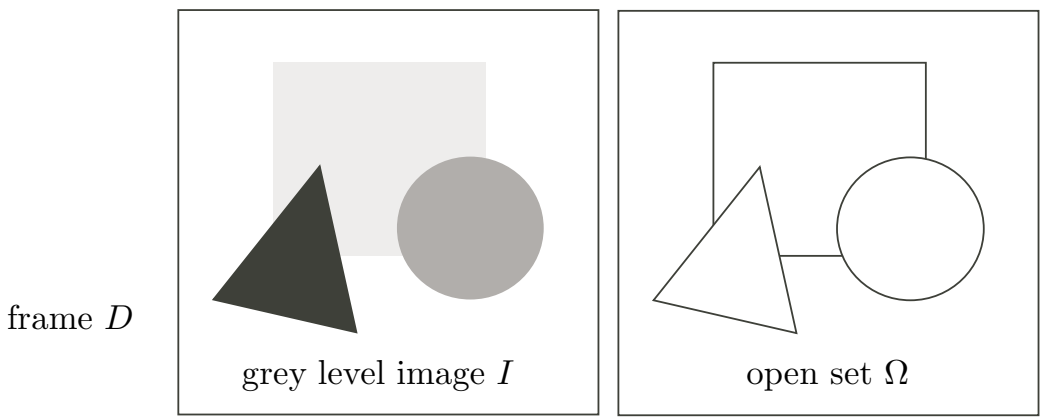

FIG. 3. Image $I$ of objects and its segmentation in an open 2-D frame $D$.

In this section we specialize to the segmentation of $N$-dimensional images where the segmentation could potentially be composed of objects of codimension greater or equal to one. To represent the set of Figure 2 as the boundary of an open set one can use the unbounded plane $\mathbf{R}^{2}$ minus all the curves and the points in Figure 2. If it is important that the open set be bounded, a fixed open frame $D$ is introduced. The open set $\Omega$ in Figure 4 is then defined as the interior of the bounded open frame $D$ minus all the curves and points used to draw the picture.

Definition 4.1. Let $D$ be a bounded open subset of $\mathbf{R}^{\mathrm{N}}$ with Lipschitzian boundary.

(i) An image in the frame $D$ is specified by a function $f \in L^{2}(D)$.

(ii) We say that $\left\{\Omega_{i}\right\}_{i \in I}$ is an open partition of $D$ if $\left\{\Omega_{i}\right\}_{i \in I}$ is a family of 


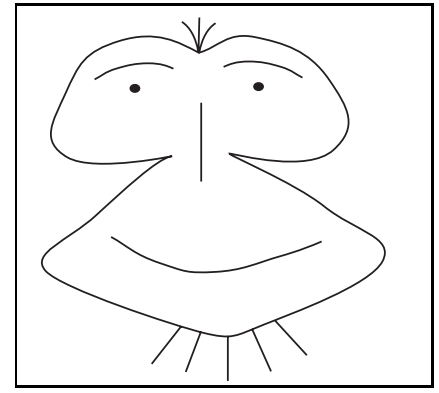

open frame $D$

FIG. 4. The 2-D strongly cracked set of Figure 2 in an open frame $D$

disjoint connected open subsets of $D$ such that

$$
\mathrm{m}_{N}\left(\cup_{i \in I} \Omega_{i}\right)=\mathrm{m}_{N}(D) \text { and } \mathrm{m}_{N}\left(\partial \cup_{i \in I} \Omega_{i}\right)=0
$$

Denote by $\mathcal{P}(D)$ the family of all such open partitions of $D$.

Given an open partition $\left\{\Omega_{i}\right\}_{i \in I}$ of $D$, associate with each $i \in I$, a function $\varphi_{i} \in H^{1}\left(\Omega_{i}\right)$. In its intuitive form the problem formulated by Mumford and Shah [21] aims at finding an open partition $P=\left\{\Omega_{i}\right\}_{i \in I}$ in $\mathcal{P}(D)$ solution of the following minimization problem

$$
\inf _{P \in \mathcal{P}(D)} \sum_{i \in I} \inf _{\varphi_{i} \in H^{1}\left(\Omega_{i}\right)} \int_{\Omega_{i}} \varepsilon\left|\nabla \varphi_{i}\right|^{2}+\left|\varphi_{i}-f\right|^{2} d x
$$

for some fixed constant $\varepsilon>0$. Observe that without the condition $\mathrm{m}_{N}\left(\cup_{i \in I} \Omega_{i}\right)=$ $\mathrm{m}_{N}(D)$ the empty set would be a solution of the problem or a phenomenon of the type discussed in Remark 3.1 and the phenomenon of Figure 1 could occur.

The question of existence requires a more specific family of open partitions or a penalization term which preserves the "length" of the interfaces in some appropriate sense:

$$
\inf _{P \in \mathcal{P}(D)} \sum_{i \in I} \inf _{\varphi_{i} \in H^{1}\left(\Omega_{i}\right)} \int_{\Omega_{i}} \varepsilon\left|\nabla \varphi_{i}\right|^{2}+\left|\varphi_{i}-f\right|^{2} d x+c H_{N-1}\left(\partial \cup_{i \in I} \Omega_{i}\right)
$$

for some $c>0$. The choice of a relaxation of the $(N-1)$-Hausdorff measure $H_{N-1}$ is critical. Here the finite perimeter of Caccioppoli reduces to the perimeter of $D$ since the characteristic function of $\cup_{i \in I} \Omega_{i}$ is almost everywhere equal to the characteristic function of $D$. In that context, the relaxation of the $(N-1)$-dimensional upper Minkowski content by Bucur and Zolésio [3] is much more interesting in view of the associated compactness Theorem 3.1.

Another way of looking at the problem would be to minimize the number $I$ of open subsets of the open partition, but this seems more difficult to formalize.

As a final remark, it is clear that the image of Figure 4 is not an $L^{2}(D)$-function and that its identification would require a sharper detection functional than the one of (4.2). 
4.2. Cracked sets without the perimeter. In this section we specialize the compact family of Theorem 3.3 to get the existence of a solution to the following minimization problem

$$
\inf _{\substack{\Omega \in \mathcal{F}(D, h, \alpha) \\ \Omega \text { open } \subset D, \mathrm{~m}_{N}(\Omega)=\mathrm{m}_{N}(D)}} \inf _{\varphi \in H^{1}(\Omega)} \int_{\Omega} \varepsilon|\nabla \varphi|^{2}+|\varphi-f|^{2} d x .
$$

This allows for open sets $\Omega$ with $H_{N-1}(\Gamma)=\infty$. The pair $(h, \alpha)$ are the control parameters of the segmentation. Recall the characterization of Theorem 3.2 (ii) which says that in each point of the boundary there exists a small open cone of uniform height and aperture which does not intersect the boundary.

TheOREm 4.1. Given a bounded open frame $D \subset \mathbf{R}^{\mathrm{N}}$ with a Lipschitzian boundary and real numbers $h, \alpha$, and $\varepsilon>0$, there exist an open subset $\Omega^{*}$ of $D$ in $\mathcal{F}(D, h, \alpha)$ such that $\mathrm{m}_{N}\left(\Omega^{*}\right)=\mathrm{m}_{N}(D)$ and $y \in H^{1}\left(\Omega^{*}\right)$ solutions of problem (4.3).

4.2.1. Technical lemmas. The proof of the existence theorems will require the following technical results.

Lemma 4.1. Given a subset $A$ of $\mathbf{R}^{\mathrm{N}}$ with nonempty boundary $\partial A$,

$$
\exists \text { an open subset } \Omega \subset \mathbf{R}^{\mathrm{N}} \text { such that } b_{A}=b_{\Omega}
$$

if and only if $d_{A}=d_{\operatorname{int} A}$ or equivalently $\bar{A}=\overline{\operatorname{int} A}$.

Proof. By definition for $\Omega$ open

$$
b_{A}=b_{\Omega} \Longleftrightarrow d_{A}=d_{\Omega} \text { and } d_{\mathrm{C} \Omega}=d_{\mathrm{C} A}
$$

which is also equivalent to

$$
\bar{A}=\bar{\Omega} \text { and } \complement \Omega=\bar{\complement} A \Longleftrightarrow \bar{A}=\bar{\Omega} \text { and } \Omega=\operatorname{int} A .
$$

Hence the necessary and sufficient condition finally reduces to $\overline{\operatorname{int} A}=\bar{A}$.

Lemma 4.2. Let $D \subset \mathbf{R}^{\mathrm{N}}$ be bounded open with Lipschitzian boundary. Then

$$
\Omega \subset D, \Gamma \neq \varnothing, \mathrm{m}_{N}(\Gamma)=0, \text { and } \mathrm{m}_{N}(\Omega)=\mathrm{m}_{N}(D) \quad \Rightarrow \overline{\operatorname{int} \Omega}=\bar{\Omega} .
$$

Proof. By contradiction. If $\overline{\operatorname{int} \Omega} \varsubsetneqq \bar{\Omega}$, there exists $x \in \bar{\Omega}$ such that $d_{\operatorname{int} \Omega}(x)=$ $\rho>0$ and hence $B(x, \rho) \subset \bar{\complement} \bar{\Omega}$. Therefore $\bar{\Omega} \cap B(x, \rho) \subset \Gamma$ and $\mathrm{m}_{N}(\bar{\Omega} \cap B(x, \rho)) \leq$ $\mathrm{m}_{N}(\Gamma)=0$. By assumption $\mathrm{m}_{N}(D)=\mathrm{m}_{N}(\Omega)=\mathrm{m}_{N}(\bar{\Omega})$ implies $\mathrm{m}_{N}(D \cap B(x, \rho))=$ $\mathrm{m}_{N}(\bar{\Omega} \cap B(x, \rho))=0$. Since $\Omega \subset D$, there exists $x \in \bar{D}$ such that $\mathrm{m}_{N}(D \cap B(x, \rho))=0$. But this is a contradiction since $D$ is an open set with Lipschitzian boundary.

4.2.2. Another compactness theorem. The compactness of the following special family of cracked sets contained in a frame is a corollary to the compactness Theorem 3.3. 
TheOREM 4.2. Let D be a bounded open subset of $\mathbf{R}^{\mathrm{N}}$ with Lipschitzian boundary and $h>0$ and $\alpha>0$ be real numbers. Consider the family

$$
\begin{aligned}
& \mathcal{F}^{c}(D, h, \alpha) \stackrel{\text { def }}{=}\left\{\begin{array}{cl}
\Omega \text { open } \subset D \text { and } \mathrm{m}_{N}(\Omega)=\mathrm{m}_{N}(D) \\
\Omega \subset \bar{D}: & \text { and } \forall x \in \Gamma, \exists d,|d|=1, \\
& \text { such that } \inf _{0<t<h} \frac{d_{\Gamma}(x+t d)}{t} \geq \alpha
\end{array}\right\} \\
& C_{b}^{c, h, \alpha}(D) \stackrel{\text { def }}{=}\left\{b_{\Omega}: \Omega \in \mathcal{F}^{c}(D, h, \alpha)\right\} .
\end{aligned}
$$

Then $C_{b}^{c, h, \alpha}(D)$ is compact in $W^{1, p}(D), 1 \leq p<\infty$.

Proof. By standard arguments and Lemma 4.2. The conclusion follows from Theorem 3.3 by adding the constraint $m_{N}\left(\Omega_{n}\right)=\mathrm{m}_{N}(D)$ which will be verified for the limit set $\Omega$ for which a subsequence of $\left\{b_{\Omega_{n}}\right\}$ converges to $b_{\Omega}$ in $W^{1,1}(D)$ and hence $\left\{\chi_{\Omega_{n}}\right\}$ converges to $\chi_{\Omega}$ in $L^{1}(D)$.

4.2.3. Proof of Theorem 4.1. Proof. [Proof of Theorem 4.1] (i) For each open $\Omega \in \mathcal{F}^{c}(D, h, \alpha)$, the problem

$$
\inf _{\varphi \in H^{1}(\Omega)} F(\Omega, \varphi), \quad F(\Omega, \varphi) \stackrel{\text { def }}{=} \int_{\Omega} \varepsilon|\nabla \varphi|^{2}+|\varphi-f|^{2} d x
$$

has a unique solution $y$ in $H^{1}(\Omega)$ since the objective function $F(\Omega, \varphi)$ is continuous and coercive on $H^{1}(\Omega)$. Define

$$
m \stackrel{\text { def }}{=} \inf _{\Omega \in \mathcal{F}^{c}(D, h, \alpha)} \inf _{\varphi \in H^{1}(\Omega)} \int_{\Omega} \varepsilon|\nabla \varphi|^{2}+|\varphi-f|^{2} d x .
$$

(ii) The minimum is finite since the objective function is positive and

$$
\forall \text { open } \Omega \in \mathcal{F}^{c}(D, h, \alpha), \quad \inf _{\varphi \in H^{1}(\Omega)} \int_{\Omega} \varepsilon|\nabla \varphi|^{2}+|\varphi-f|^{2} d x \leq \int_{\Omega}|f|^{2} d x
$$

by choosing $\varphi=0$. Let $\left\{\Omega_{n}\right\}$ be a minimizing sequence of open subsets of $D$ in $\mathcal{F}^{c}(D, h, \alpha)$ and for each $n$ let $y_{n} \in H^{1}\left(\Omega_{n}\right)$ be the minimizing element of $F\left(\Omega_{n}, \varphi\right)$ over $H^{1}\left(\Omega_{n}\right)$. Therefore,

$$
\begin{gathered}
\int_{\Omega_{n}} \varepsilon\left|\nabla y_{n}\right|^{2}+\left|y_{n}-f\right|^{2} d x \rightarrow m \\
\Rightarrow \exists c>0 \text { such that } \forall n, \quad \int_{\Omega_{n}} \varepsilon\left|\nabla y_{n}\right|^{2}+\left|y_{n}-f\right|^{2} d x \leq c .
\end{gathered}
$$

By coercivity the sequence $\left\{y_{n}\right\}$ is uniformly bounded in $H^{1}\left(\Omega_{n}\right)$, that is there exists a constant $c>0$ such that

$$
\forall n, \quad\left\|y_{n}\right\|_{L^{2}\left(\Omega_{n}\right)} \leq c \text { and }\left\|\nabla y_{n}\right\|_{L^{2}\left(\Omega_{n}\right)} \leq c .
$$

By Theorem 4.2 there exists a subsequence of $\left\{\Omega_{n}\right\}$ and an open set $\Omega \in \mathcal{F}^{c}(D, h, \alpha)$ such that $b_{\Omega_{n}} \rightarrow b_{\Omega}$ in $H^{1}(D)$ and $C(\bar{D})$. In particular $d_{\complement \Omega_{n}} \rightarrow d_{\complement \Omega}$ in $C(\bar{D})$. By the 
compactivorous property (cf. [11], Theorem 2.4 (iii), p. 162),

$\forall K$ compact $\subset$ int $\Omega, \quad \exists N$ such that $\forall n \geq N, \quad K \subset \Omega_{n}$.

Moreover,

$$
\begin{gathered}
\chi_{\Omega_{n}} \rightarrow \chi_{\Omega} \in L^{2}(D) \quad \Rightarrow \chi_{\Omega_{n}} \rightarrow \chi_{\Omega} \in L^{\infty}(D) \text {-weak* } \\
\Rightarrow f \chi_{\Omega_{n}} \rightarrow f \chi_{\Omega} \in L^{2}(D) \text {-weak. }
\end{gathered}
$$

Define the distributions

$$
\begin{gathered}
<\tilde{y}_{n}, \varphi>\stackrel{\text { def }}{=} \int_{\Omega_{n}} y_{n} \varphi d x, \quad \forall \varphi \in \mathcal{D}(D) \\
<\widetilde{\nabla y_{n}}, \Phi>\stackrel{\text { def }}{=} \int_{\Omega_{n}} \nabla y_{n} \cdot \Phi d x, \quad \forall \Phi \in \mathcal{D}(D)^{N} \\
<\nabla \tilde{y}_{n}, \Phi>=-\int_{D} \tilde{y}_{n} \operatorname{div} \Phi d x, \quad \forall \Phi \in \mathcal{D}(D)^{N} .
\end{gathered}
$$

It is readily seen that we can identify $\tilde{y}_{n}$ and $\widetilde{\nabla y_{n}}$ with the extensions of $y_{n}$ and $\nabla y_{n}$ by zero from $\Omega_{n}$ to $D$. As a result there exist subsequences, still denoted $\left\{\tilde{y}_{n}\right\}$ and $\left\{\widetilde{\nabla y_{n}}\right\}$, and $\tilde{y} \in L^{2}(D)$ and $Y \in L^{2}(D)^{N}$ such that $\tilde{y}_{n} \rightarrow \tilde{y}$ in $L^{2}(D)$-weak and $\widetilde{\nabla y_{n}} \rightarrow Y$ in $L^{2}(D)^{N}$-weak.

By the compactivorous property, for all $\Phi \in \mathcal{D}(\Omega)$, there exists $N$ such that

$$
\forall n>N, \quad \operatorname{supp} \Phi \subset \Omega_{n} \quad \Rightarrow \Phi \in \mathcal{D}\left(\Omega_{n}\right) .
$$

Therefore, for all $n>N$,

$$
<\nabla \tilde{y}_{n}-\widetilde{\nabla y_{n}}, \Phi>=-\int_{\Omega_{n}} y_{n} \operatorname{div} \Phi d x-\int_{\Omega_{n}} \nabla y_{n} \cdot \Phi d x=0
$$

since $y_{n} \in H^{1}\left(\Omega_{n}\right)$. But $\mathcal{D}\left(\Omega_{n}\right) \subset \mathcal{D}(D)$ and by letting $n$ go to infinity,

$$
\begin{gathered}
0=-\int_{\Omega_{n}} y_{n} \operatorname{div} \Phi d x-\int_{\Omega_{n}} \nabla y_{n} \cdot \Phi d x \rightarrow-\int_{D} \tilde{y} \operatorname{div} \Phi d x-\int_{D} Y \cdot \Phi d x \\
\Rightarrow \forall \Phi \in \mathcal{D}(\Omega), \quad \int_{D} \tilde{y} \operatorname{div} \Phi d x+\int_{D} Y \cdot \Phi d x=0 .
\end{gathered}
$$

Define the new distribution

$$
<y, \varphi>\stackrel{\text { def }}{=} \int_{\Omega} \tilde{y} \varphi d x, \quad \forall \varphi \in \mathcal{D}(\Omega)
$$

It is easy to check that $y \in L^{2}(\Omega)$ and hence

$$
\begin{gathered}
\forall \Phi \in \mathcal{D}(\Omega), \quad 0=\int_{D} \tilde{y} \operatorname{div} \Phi d x+\int_{D} Y \cdot \Phi d x=\int_{\Omega} \tilde{y} \operatorname{div} \Phi d x+\int_{\Omega} Y \cdot \Phi d x \\
\Rightarrow \forall \Phi \in \mathcal{D}(\Omega), \quad<\nabla y, \Phi>=-\int_{\Omega} \tilde{y} \operatorname{div} \Phi d x=\int_{\Omega} Y \cdot \Phi d x \\
\Rightarrow \nabla y=\left.Y\right|_{\Omega} \in L^{2}(\Omega) \quad \Rightarrow y \in H^{1}(\Omega) .
\end{gathered}
$$


(iv) Coming back to our objective function

$$
\begin{aligned}
\inf _{\varphi \in H^{1}\left(\Omega_{n}\right)} \int_{\Omega_{n}} \varepsilon|\nabla \varphi|^{2}+|\varphi-f|^{2} d x & =\int_{\Omega_{n}} \varepsilon\left|\nabla y_{n}\right|^{2}+\left|y_{n}-f\right|^{2} d x \\
& =\int_{D} \varepsilon\left|\widetilde{\nabla y_{n}}\right|^{2}+\left|\tilde{y}_{n}-f \chi_{\Omega_{n}}\right|^{2} d x .
\end{aligned}
$$

By convexity and continuity of the objective function with respect to the pair $\left(\tilde{y}_{n}-\right.$ $\left.f \chi_{\Omega_{n}}, \widetilde{\nabla y_{n}}\right)$ in $L^{2}(D) \times L^{2}(D)^{N}$ and the fact that, from $(4.6),\left(\tilde{y}_{n}-f \chi_{\Omega_{n}}, \widetilde{\nabla y_{n}}\right) \rightarrow$ $\left(\tilde{y}-f \chi_{\Omega}, Y\right)$ in $L^{2}(D) \times L^{2}(D)^{N}$-weak,

$$
\begin{aligned}
\int_{D} \varepsilon|Y|^{2}+\left|\tilde{y}-f \chi_{\Omega}\right|^{2} d x \leq & \liminf _{n \rightarrow \infty} \int_{D} \varepsilon\left|\widetilde{\nabla y_{n}}\right|^{2}+\left|\tilde{y}_{n}-f \chi_{\Omega_{n}}\right|^{2} d x \\
& =\liminf _{n \rightarrow \infty} \int_{\Omega_{n}} \varepsilon\left|\nabla y_{n}\right|^{2}+\left|y_{n}-f\right|^{2} d x=m \\
\Rightarrow \int_{\Omega} \varepsilon|\nabla y|^{2}+|y-f|^{2} d x & =\int_{D} \varepsilon|\nabla y|^{2}+\left|y-f \chi_{\Omega}\right|^{2} d x \leq m .
\end{aligned}
$$

By definition of the minimum we have the equality and there exist an open set $\Omega \in$ $\mathcal{F}^{c}(D, h, \alpha)$ and $y \in H^{1}(\Omega)$ solution of the segmentation problem.

4.3. Existence of a cracked set with minimum density perimeter. Theorem 4.1 gives an existence result for the family $\mathcal{F}(D, h, \alpha)$ of open sets $\Omega$ such that $\mathrm{m}_{N}(\Omega)=\mathrm{m}_{N}(D)$ without constraint on the "perimeter" of $\Omega$. Denote by $\mathcal{F}^{*}(D, h, \alpha)$ the set of solutions to problem (4.3). In general, the perimeter can be infinite as can be seen from the following example.

EXAMPLE 4.1. The function $f: D \rightarrow \mathbf{R}$ is defined as follows

$$
f(x)= \begin{cases}1, & \text { if } x \in \Omega_{1} \\ 0, & \text { if } x \in \Omega_{2}\end{cases}
$$

where $D=\{(x, y):-2<x<3,-1<y<3\}, \Omega=\Omega_{1} \cup \Omega_{2}, \Omega_{2}=D \backslash \overline{\Omega_{1}}$, and the open set $\Omega_{1}$ is constructed below (see Figure 5). The set $\Omega$ with $y=f$ is a solution of problem (4.3) with infinite perimeter.

The set $\Omega_{1}$ is a two-dimensional example constructed by Nicolas Doyon ${ }^{11}$ of an open domain satisfying the uniform cusp condition of [11] for the function $h(\theta)=\theta^{\alpha}$, $0<\alpha<1$. It can easily be generalized to an $N$-dimensional example. Consider the open domain $\Omega_{1}$ in $\mathbf{R}^{2}$

$$
\begin{aligned}
\Omega_{1} \stackrel{\text { def }}{=} & \{(x, y):-1<x \leq 0 \text { and } 0<y<2\} \\
& \cap\{(x, y): 0<x<1 \text { and } f(x)<y<2\} \\
& \cap\{(x, y): 1 \leq x<2 \text { and } 0<y<2\},
\end{aligned}
$$

\footnotetext{
${ }^{11}$ Département de Mathématiques et de statistique, Université de Montréal.
} 
where $f:[0,1] \rightarrow \mathbf{R}$ is defined as follows

$$
f(x) \stackrel{\text { def }}{=} \sum_{k=0}^{\infty} f_{k}(x), \quad f_{k}:\left[1-\frac{1}{2^{k}}, 1-\frac{1}{2^{k+1}}\right] \rightarrow \mathbf{R} .
$$

Associate with $0<\alpha<1$ and $k \geq 0$ the even integer $\eta_{k}=2\left[\left(2^{k+1}\right)^{\frac{\alpha}{1-\alpha}}\right]$, where $[\beta]$ is

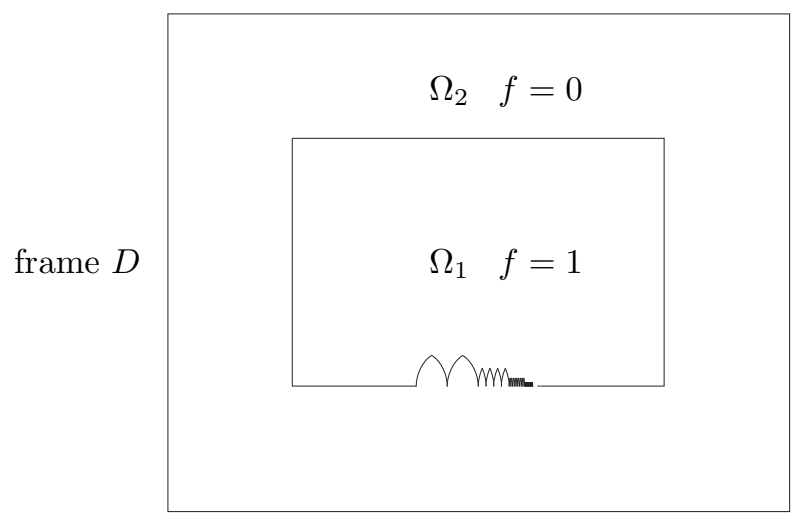

FIG. 5. The two open components $\Omega_{1}$ and $\Omega_{2}$ of the open domain $\Omega$ for $N=2$.

the smallest integer greater or equal to $\beta$. Assume that for each $k \geq 0$

$$
\begin{gathered}
f_{k} \stackrel{\text { def }}{=} \sum_{j=1}^{\eta_{k} / 2} g_{k, j} \quad g_{k, j}:\left[x_{k, j-1}, x_{k, j-1}+\delta_{k}\right] \rightarrow \mathbf{R} \\
x_{k, j} \stackrel{\text { def }}{=} 1-\frac{1}{2^{k}}+(j-1) 2 \delta_{k}, 1 \leq j \leq \eta_{k} / 2, \quad \delta_{k} \stackrel{\text { def }}{=} \frac{1}{\eta_{k} 2^{k+1}}
\end{gathered}
$$

and that the function $g_{k, j}$ is given by the expression

$$
g_{k, j}(x) \stackrel{\text { def }}{=} \begin{cases}0, & 0 \leq x<x_{k, j-1} \\ \left(x-x_{k, j-1}\right)^{\alpha}, & x_{k, j-1} \leq x \leq x_{k, j-1}+\delta_{k} \\ \left(2 x_{k, j-1}-x\right)^{\alpha}, & x_{k, j-1}+\delta_{k} \leq x \leq x_{k, j-1}+2 \delta_{k} \\ 0, & x>x_{k, j-1}+2 \delta_{k} .\end{cases}
$$

Note that

$$
g_{k, j}\left(x_{k, j-1}+\delta_{k}\right)=\left(\delta_{k}\right)^{\alpha}
$$

is independent of $j$ and is the maximum of the function $g_{k, j}$.

The uniform cusp property is verified for $\rho=1 / 8, \lambda=h(\rho)$, and $h(\theta)=\theta^{\alpha}$. The boundary of $\Omega_{1}$ is made up of straight lines of total length 9 plus the length of the curve

$$
\begin{gathered}
C \stackrel{\text { def }}{=}\{(x, f(x)): 0 \leq x<1\}, \quad C=\cup_{k=0}^{\infty} C_{k}, \quad C_{k}=\cup_{j=1}^{\eta_{k} / 2} C_{k, j} \\
C_{k, j} \stackrel{\text { def }}{=}\left\{(x, f(x)): x_{k, j-1} \leq x<x_{k, j-1}+\delta_{k}\right\} .
\end{gathered}
$$


The length of the curve $C_{k, j}$ is bounded below by

$$
\begin{gathered}
H_{N-1}\left(C_{k, j}\right) \geq 2 \sqrt{\left(\delta_{k}\right)^{2}+\left(\delta_{k}\right)^{2 \alpha}} \geq 2\left(\delta_{k}\right)^{\alpha} \Rightarrow H_{N-1}\left(C_{k}\right) \geq \sum_{j=1}^{\eta_{k} / 2} H_{N-1}\left(C_{k, j}\right) \\
H_{N-1}\left(C_{k}\right) \geq \frac{\eta_{k}}{2} 2\left(\delta_{k}\right)^{\alpha}=\eta_{k}\left(\delta_{k}\right)^{\alpha}=\eta_{k}\left(\frac{1}{\eta_{k} 2^{k+1}}\right)^{\alpha}=\eta_{k}{ }^{1-\alpha}\left(\frac{1}{2^{k+1}}\right)^{\alpha} \\
H_{N-1}\left(C_{k}\right) \geq \eta_{k}{ }^{1-\alpha}\left(\frac{1}{2^{k+1}}\right)^{\alpha}=\left(2\left[\left(2^{k+1}\right)^{\frac{\alpha}{1-\alpha}}\right]\right)^{1-\alpha}\left(\frac{1}{2^{k+1}}\right)^{\alpha} \\
\geq 2^{1-\alpha}\left(2^{k+1}\right)^{\alpha}\left(\frac{1}{2^{k+1}}\right)^{\alpha}=2^{1-\alpha} \\
\Rightarrow H_{N-1}(C)=\sum_{k=0}^{\infty} H_{N-1}\left(C_{k}\right) \geq+\infty 2^{1-\alpha}=+\infty
\end{gathered}
$$

When at least one solution has a bounded perimeter, it is possible to show that there is one that minimizes the $h$-density perimeter.

TheOREm 4.3. Assume that the assumptions of Theorem 4.1 are verified. There exists an $\Omega^{*}$ in $\mathcal{F}^{*}(D, h, \alpha)$ which minimizes the $h$-density perimeter.

Proof. If for all $\Omega$ in $\mathcal{F}^{*}(D, h, \alpha)$ the $h$-density perimeter is $+\infty$ the theorem is true. If for some $\Omega \in \mathcal{F}^{*}(D, h, \alpha), P_{h}(\Gamma) \leq c$, then there exists a sequence $\left\{\Omega_{n}\right\}$ in $\mathcal{F}^{*}(D, h, \alpha)$ such that

$$
P_{h}\left(\Gamma_{n}\right) \rightarrow \inf _{\Omega \in \mathcal{F}^{*}(D, h, \alpha)} P_{h}(\Gamma)
$$

By the compactness Theorems 3.1 and 4.2 , there exist a subsequence and $\Omega^{*}, \Gamma^{*} \neq \varnothing$, such that $b_{\Omega_{n}} \rightarrow b_{\Omega^{*}}$ in $W^{1, p}(D), \Omega^{*} \in \mathcal{F}(D, h, \alpha), \mathrm{m}_{N}(\Omega)=\mathrm{m}_{N}(D)$, and

$$
P_{h}\left(\Gamma^{*}\right) \leq \liminf _{n \rightarrow \infty} P_{h}\left(\Gamma_{n}\right) \leq c .
$$

Finally by going back to the proof of Theorem 4.1 and using the fact that all the $\Omega_{n}$ 's are already minimizers in $\mathcal{F}^{*}(D, h, \alpha)$, it can be shown that $\Omega^{*}$ is indeed one of the minimizers in the set $\mathcal{F}^{*}(D, h, \alpha)$.

\subsection{Uniform bound or penalization term in the objective function on} the density perimeter. To complete the results on the segmentation problem, we turn to the existence of a segmentation for a family of sets with a uniform bound or with a penalization term in the objective function on the $h$-density perimeter.

THEOREM 4.4. Given a bounded open frame $D \subset \mathbf{R}^{\mathrm{N}}$ with a Lipschitzian boundary and real numbers $h>0$ and $c>0^{12}$, there exists an open subset $\Omega^{*}$ of $D, \Gamma^{*} \neq \varnothing$,

\footnotetext{
${ }^{12}$ Note that the constant $c$ must be large enough to take into account the contribution of the boundary of $D$.
} 
with finite h-density parameter such that $\mathrm{m}_{N}\left(\Omega^{*}\right)=\mathrm{m}_{N}(D)\left(P_{h}\left(\Gamma^{*}\right) \leq c\right.$ for (4.8)), and $y \in H^{1}\left(\Omega^{*}\right)$ solutions of the respective problems

$$
\frac{\inf _{\substack{\Omega \text { open } \subset D, P_{h}(\Gamma) \leq c \\ \mathrm{~m}_{N}(\Omega)=\mathrm{m}_{N}(D)}} \inf _{\varphi \in H^{1}(\Omega)} \int_{\Omega} \varepsilon|\nabla \varphi|^{2}+|\varphi-f|^{2} d x}{\inf _{\substack{\Omega \text { open } \subset D \\ \mathrm{~m}_{N}(\Omega)=\mathrm{m}_{N}(D)}} \inf _{\varphi \in H^{1}(\Omega)} \int_{\Omega} \varepsilon|\nabla \varphi|^{2}+|\varphi-f|^{2} d x+c P_{h}(\Gamma) .}
$$

Proof. The proof for the objective function (4.8) is exactly the same as the one of Theorem 4.1. It uses Lemma 4.2 to show that the minimizing set has a thin boundary and the compactness Theorem 3.1. The proof for the objective function (4.9) uses the fact that there is a minimizing sequence for which the $h$-density perimeter is uniformly bounded and the lower semicontinuity of the density perimeter in the $W^{1, p}$-topology given by Corollary 3.1.

Problem (4.9) was the one originally considered in [3]. The above two identification problems can be further specialized to the family of cracked sets $\mathcal{F}(D, h, \alpha)$.

Corollary 4.1. Given a bounded open frame $D \subset \mathbf{R}^{\mathrm{N}}$ with a Lipschitzian boundary and real numbers $h>0, \alpha>0$ and $c>00^{13}$, there exists an open subset $\Omega^{*}$ of $D$ in $\mathcal{F}(D, h, \alpha)$ such that $\mathrm{m}_{N}\left(\Omega^{*}\right)=\mathrm{m}_{N}(D)\left(P_{h}\left(\Gamma^{*}\right) \leq c\right.$ for (4.10)), and $y \in H^{1}\left(\Omega^{*}\right)$ solutions of the problem

$$
\begin{array}{|l}
\inf _{\substack{\Omega \text { open } \subset D, \Omega \in \mathcal{F}(D, h, \alpha) \\
P_{h}(\Gamma) \leq c, \mathrm{~m}_{N}(\Omega)=\mathrm{m}_{N}(D)}} \inf _{\varphi \in H^{1}(\Omega)} \int_{\Omega} \varepsilon|\nabla \varphi|^{2}+|\varphi-f|^{2} d x \\
\inf _{\substack{\Omega \text { open } \subset D, \Omega \in \mathcal{F}(D, h, \alpha) \\
\mathrm{m}_{N}(\Omega)=\mathrm{m}_{N}(D)}} \inf _{\varphi \in H^{1}(\Omega)} \int_{\Omega} \varepsilon|\nabla \varphi|^{2}+|\varphi-f|^{2} d x+c P_{h}(\Gamma) . \\
\hline
\end{array}
$$

Proof. Since the minimizing sequence $\left\{b_{\Omega_{n}}\right\}$ constructed in the proof of Theorem 4.1 strongly converges to $b_{\Omega^{*}}$ in $W^{1, p}(D)$ for all $p, 1 \leq p<\infty$, from property (3.4) in Theorem 3.1, we have

$$
P_{h}\left(\Gamma^{*}\right) \leq \liminf _{n \rightarrow \infty} P_{h}\left(\Gamma_{n}\right) \leq c
$$

and the optimal $\Omega^{*}$ constructed in the proof of the theorem satisfies the additional constraint on the density perimeter.

\footnotetext{
${ }^{13}$ Note that the constant $c$ must be large enough to take into account the contribution of the boundary of $D$.
} 
[1] J. Blat and J. M. Morel, Elliptic Problems in Image Segmentation and their Relation to Fracture, In: Recent Advances in Nonlinear Elliptic and Parabolic Problems, pp. 216-228, 1989.

[2] K. B. Broberg, Cracks and fracture, Academic Press, 1999.

[3] D. Bucur And J.-P. ZolÉsio, Free boundary problems and density perimeter, J. Differential Equations 126(1996), pp. 224-243.

[4] D. Bucur And J.-P. ZolÉsio, Boundary optimization under pseudo curvature constraint, Annali della Scuola Normale Superiore di Pisa, Sc. Fis e Mat, Ser IV, XXIII, Fasc. 4 (1996), pp. 681-699.

[5] G. Dal Maso, J. M. Morel, and S. Solimini, A variational method in image segmentation: existence and approximation results, Ref. S.I.S.S.A. 48 M, April 1989, S.I.S.S.A., Srada Costeria 11-34014 Trieste Italy.

[6] M. C. Delfour, N. Doyon, And J.-P. ZolÉsio, Uniform cusp property, boundary integral, and compactness for shape optimization, In: System Modeling and Optimization, J. Cagnol and J.-P. Zolésio, eds, pp. 25-40, Kluwer Academic Publishers 2004.

[7] M.C. Delfour, N. Doyon, And J.-P. Zolésio, Extension of the uniform cusp property in shape optimization, In: Control of Partial Differential Equations, G. Leugering, O. Imanuvilov, R. Triggiani, and B. Zhang, eds. Lectures Notes in Pure and Applied Mathematics, Marcel Dekker, to appear (2004).

[8] M.C. Delfour, N. Doyon, And J.-P. Zolésio, The uniform fat segment and cusp properties in shape optimization, In: Control and Boundary Analysis, J. Cagnol and J.-P. Zolésio, eds, pp. 85-96, Marcel Dekker 2004.

[9] M. C. Delfour And J.-P. ZolÉSIO, Shape analysis via oriented distance functions, J. Funct. Anal. 123:1(1994), pp. 129-201.

[10] M. C. Delfour And J.-P. Zolésio, Shape analysis via distance functions: local theory, In: Boundaries, interfaces, and transitions, pp. 91-123, CRM Proc. Lecture Notes, 13, Amer. Math. Soc., Providence, RI, 1998.

[11] M. C. Delfour And J.-P. Zolésio, Shapes and Geometries: Analysis, Differential Calculus and Optimization, SIAM series on Advances in Design and Control, Society for Industrial and Applied Mathematics, Philadelphia, USA 2001.

[12] M. C. Delfour And J.-P. ZolÉsio, Oriented distance function and its evolution equation for initial sets with thin boundary, SIAM J. on Control and Optim., 42:6(2004), pp. 2286-2304.

[13] M. C. Delfour And J.-P. ZolÉSIO, Shape identification via metrics constructed from the oriented distance function, Control and Cybernetics, accepted 2004.

[14] C. Dellacherie, Ensembles analytiques, capacités, mesures de Hausdorff, Springer-Verlag, Berlin, Heidelberg, New York 1972.

[15] S. Geman and D. Geman, Stochastic relaxation, Gibbs distributions and the bayesian restoration of images, IEEE Trans. PAMI 6(1984), pp. 721-741.

[16] D. Geman, S. Geman, And P. Dong, Boundary detection by constrained optimization, IEEE Trans. PAMI 12:7(1990), pp. 609-628.

[17] S. R. Kulkarni, S. Mitter, and T.J. Richardson, An existence theorem and lattice approximations for a variational problem arising in computer vision, Signal processing, Part I, L. Auslander, T. Kailath, and S. Mitter, eds, pp. 189-210, IMA Vol. Math. Appl., 22, Springer, New York, 1990.

[18] S. R. Kulkarni, S. Mitter, T. J. Richardson, and J. N. Tsitsiklis, Local versus nonlocal computation of length of digitized curves, Foundations of software technology and theoretical computer science (Bombay, 1993), pp. 94-103, Lecture Notes in Comput. 
Sci., 761, Springer, Berlin 1993.

[19] J. M. Morel And S. Solimini, Variational Methods in Image Segmentation, Birkhäuser, 1995.

[20] D. Mumford, Pattern theory: a unifying perspective, In: First European Congress of Mathematics, J. Anthony et al., eds, pp. 187-224, Springer-Verlag, 1994.

[21] D. Mumford and J. Shah, Optimal approximations by piecewise smooth functions and associated variational problems, Comm. on Pure and Appl. Math., XLII(1989), pp. $577-685$.

[22] T. J. Richardson, Scale independent piecewise smooth segmentation of images via variational methods, Report CICS-TH-194, Center for Intelligent Control Systems, Massachusetts Institute of Technology, Cambridge, Mass., February 1990.

[23] T. J. Richardson and S. K. Mitter, Approximation computation and distortion in the variational formulation, In: Geometric-Driven Diffusion in Computer Vision. Bart M. ter Haar Romeny, editor, chapter 8, pp. 169-190, Kluwer Academic Publishers, Dordrecht/Boston/London, 1994.

[24] T. J. Richardson and S. K. Mitter, A variational formulation-based edge focussing algorithm, Academy Proceedings in Engineering Sciences, Indian Acad. Sci. 22:4(1997), pp. 553-574.

[25] A. Rosenfeld and J. L. Pfaltz, Distance functions on digital pictures, Pattern Recognition, 1:1(1968), pp. 33-61.

[26] J. SERRA, Image analysis and mathematical morphology, English version revised by Noel Cressie. Academic Press, Inc. [Harcourt Brace Jovanovich, Publishers], London, 1982, reprinted 1984.

[27] J. Shaн, Segmentation by nonlinear diffusion, In: Conference on Computer Vision and Pattern Recognition, pp. 202-207, June 1991.

[28] Bart M. ter HaAR Romeny, editor, Geometric-Driven Diffusion in Computer Vision Kluwer Academic Publishers, Dordrecht/Boston/London, 1994.

[29] S. Yokoi, J.-I. Toriwaki, And T. Fukumura, On Generalized Distance Transformation of Discretized Pictures, IEEE PAMI 3(1981), pp. 424-443. 